\title{
FIXED POINTS AND STABLE SUBGROUPS OF ALGEBRAIC GROUP AUTOMORPHISMS ${ }^{1}$
}

\author{
D. J. WINTER
}

1. Introduction. In this paper, we study the fixed point sets and stable subgroups of automorphisms of a connected algebraic linear group over an algebraically closed field of arbitrary characteristic. [7] contains a description of most of the results of this paper, and some material in [7] is essential for the present paper. Many of the results discussed in the present paper were proved at characteristic 0 by Borel-Mostow, Jacobson and Mostow (see [1], [5], [6]).

In $\$ 3$ it is essentially shown that if $\sigma_{1}, \sigma_{2}$ are "semisimple" automorphisms of a connected linear algebraic group $G$ which differ by an inner automorphism of $G$, and if $H_{i}$ is a Cartan subgroup of the connected component of the subgroup of fixed points of $\sigma_{i}(i=1,2)$, then there is an inner automorphism Int $g$ of $G$ such that $H_{1}$ Int $g=H_{2}$ and Int $g^{-1} \circ \sigma_{1} \circ$ Int $H_{1} \circ$ Int $g=\sigma_{2} \circ$ Int $H_{2}$. This is used (see $\$ 4$ ) to establish that a semisimple automorphism of a connected algebraic linear group $G$ stabilizes some Borel subgroup of $G$ and some Cartan subgroup of $G$; and that a semisimple automorphism of a connected semisimple algebraic group $G$ keeps fixed some regular element of $G$.

2. Preliminaries. Throughout this paper, $G$ is a connected linear algebraic group over an algebraically closed field of arbitrary characteristic 0 or $p$. We do not distinguish between $G$ and birational isomorphic images of $G$. Some familiarity with the general structure theory of algebraic groups is assumed in the discussion (see [3]).

A (birational) automorphism $\sigma$ of $G$ is algebraic if $G$ can be birationally identified with a closed normal subgroup of an algebraic linear group $K$ over $F$ which contains an element $s$ such that $\sigma=\operatorname{Int}_{G} s$ (Int ${ }_{G} s$ denotes the automorphism of $G$ sending an element $g$ of $G$ into $s^{-1} g s$ ). Such a $\sigma$ is said to be semisimple (unipotent) if for some such $K$ and $s, s$ is semisimple (unipotent). (This terminology is equivalent to that of [7], as is noted in [7].)

An algebraic automorphism $\sigma$ has a unique decomposition $\sigma=\sigma_{s} \sigma_{u}$ where $\sigma_{s}, \sigma_{u}$ are commuting algebraic automorphisms which are

Received by the editors August 13, 1966.

1 This paper was written while the author was supported by the Army Research Office, Durham. It is a reformulation of material contained in a dissertation directed by Professor George Seligman, presented for the degree of Doctor of Philosophy at Yale University, and written on a National Science Foundation Fellowship. 
respectively semisimple and unipotent. Every (birational) automorphism of a connected semisimple algebraic linear group over $F$ is algebraic. (The above facts are proved in [7].)

The Zariski-connected component of the identity of a subgroup $S$ of $G$ is denoted by $S_{0}$. The set of fixed points of an automorphism $\sigma$ of $G$ is denoted by $F_{G}(\sigma)$ (this is a slight departure from the terminology of [7]). If $K$ is an algebraic linear group containing $G$ as a closed normal subgroup and if $g, h$ are elements of $K$, we use the following notation: $g^{h}=h^{-1} g h ; C_{G}(h)=\{g$ in $G \mid g h=h g\} ; h_{s}, h_{u}$ denote respectively the semisimple and unipotent parts of $h$.

3. Cartan subgroups of $F(\sigma)_{0}$. In this section it is in effect shown that if $\sigma$ is a semisimple automorphism of $G$, then properties of a Cartan subgroup of $F(\sigma)_{0}$ relative to the action of $G$ on itself given by $g: x \rightarrow \sigma\left(g^{-1}\right) x g$ are roughly the same as properties of a Cartan subgroup of $G$ relative to the action of $G$ on itself given by $g: x \rightarrow g^{-1} x g$. This is then used to establish the following theorem, which is needed for the material discussed in the next section:

THEOREM 1. Let $\sigma$ and $\tau$ be semisimple elements of a linear algebraic group $K$ containing $G$ as a closed normal subgroup. Suppose that $\sigma G=\tau G$. Let $H$ be a Cartan subgroup of $C_{G}(\sigma)_{0}, L$ a Cartan subgroup of $C_{G}(\tau)_{0}$. Then there exists $g$ in $G$ such that $H^{o}=L$ and $(\sigma H)^{o}=\tau L$.

Lemma 1. Let $K$ be an algebraic group. Let $G, R$ be closed connected normal subgroups of $K$ with $R \subseteq G$ and $R$ solvable. Let $\sigma$ be a semisimple element of $K$ such that $g^{\sigma} R=g R$ for $g$ in $G$. Then $G=C_{G}(\sigma)_{0} R$.

Proof. Suppose first that $R$ is a torus. Let $B$ be a Borel subgroup of $G . B \supseteq R$ (since $B R$ is a solvable connected subgroup of $G$ ) and $B$ has the form $B=T U$ where $T$ is a maximal torus of $B$ and $U$ is a closed normal unipotent subgroup of $B$ (see $[3, \S 6-06]$ ). As a normal torus in $B, R$ is central in $B$ (see $[3, \S 4-07]$ ) and hence is contained in $T$. Thus $T^{\sigma}=T$. And $\sigma \mid U=i d_{U}$ (since for $u$ in $U, u^{\sigma}=u s$ for some $s$ in $R$; and $u s=s u$ since $R$ is central in $B$, so that $s=1$ since $u^{\sigma}$ is semisimple). We claim that $T=C_{T}(\sigma) R$ (it then follows that $\left.B \subseteq C_{G}(\sigma) R\right)$. Suppose first that $\sigma$ has finite order $k$. Let $\mathfrak{g}=\langle\sigma\rangle$ be the cyclic group generated by $\sigma$ and let $l$ be an integer with $(k, l)=1$. For any torus $S$, we define $S_{l}=\left\{s\right.$ in $\left.S \mid s^{l}=1\right\}$. Then $T_{l}$ and $R_{l}$ are $\mathfrak{g}$-stable. And for $t$ in $T_{l}, \tau$ in $\mathfrak{g}$, there exists a unique $s_{\tau}$ in $R_{l}$ such that $t^{\tau}=t s_{\tau}$. The mapping $s_{\tau}$ from $g$ into $R_{l}$ is a 1-cocycle, as is easily checked. Since $(k, l)=1, H^{1}\left(\mathfrak{g}, R_{l}\right)=\{0\}$ and there exists $c$ in $R_{l}$ such that $c\left(c^{-1}\right)^{\tau}=s_{\tau}$ for $\tau$ in $g$. For such a $c,(t c)^{\sigma}=t s_{\sigma} c^{\sigma}=t c$. Thus 
$T_{l} \subseteq C_{G}(\sigma) R$. Since $\bigcup_{(k, l)=1} T_{l}$ is dense in $T$ (see $[3, \S 6-13]$ ), it follows that $T \subseteq C_{G}(\sigma) R$. Thus $B \subseteq C_{G}(\sigma) R$. Since every element of $G$ is contained in some Borel subgroup of $G$, it follows that $G \subseteq C_{G}(\sigma) R$. Thus $G \subseteq C_{G}(\sigma)_{0} R$.

If $\sigma$ is not finite, let $A$ be the closed subgroup of $K$ generated by $\sigma$. Then $A=\bigcup_{i=1}^{m} A_{0} \sigma^{i}$ where $m=\left(A: A_{0}\right)$. Choose $a_{j}$ in $A_{0}$ such that $\left\langle a_{j}\right\rangle \subseteq\left\langle a_{j+1}\right\rangle$ for all $j, U\left\langle a_{j}\right\rangle$ is dense in $A_{0}$, and $a_{j}$ has finite order relatively prime to $m$ for all $j$ (see $[3, \S 6-13]$ ). Choose $a$ in $A_{0}$ such that $a^{m}=\sigma^{m}$ (this is possible since $A_{0}$ is a torus). Let $\tau=\sigma a^{-1}$. Then $\tau$ has order $m$ and the groups $g_{j}$ generated by $\tau$ and $a_{j}$ are finite cyclic groups (since $A$ is abelian and $a_{j}$ has finite order relatively prime to $m)$. Since $A=\bigcup_{i=1}^{m} A_{0} \tau^{i}, \bigcup_{\mathfrak{g}_{j}}$ is dense in $A$. Choose $j$ such that $C_{G}\left(\mathfrak{g}_{j}\right)$ $=C_{G}(A)=C_{G}(\sigma)$. Since $\mathfrak{g}_{j}$ is generated by a single semisimple element of $K$ of finite order, we have $G \subseteq C_{G}\left(\mathfrak{g}_{j}\right)_{0} R=C_{G}(\sigma)_{0} R$ by previous work.

We now consider the general case, $R$ solvable. Let $V$ be the (closed characteristic connected) subgroup of unipotent elements of $R$ (see $[3, \S 6-06])$. Let $\bar{\sigma}$ be the automorphism of $\bar{G}=G / V$ induced by $\sigma$. Since $\bar{R}=R / V$ is a normal torus in $\bar{G}$, we have $\bar{G}=C_{\bar{G}}(\bar{\sigma}) \bar{R}$. To show that $G=C_{G}(\sigma) R$, it therefore suffices to show that if $g$ in $G$ satisfies $g^{\sigma} V=g V$, then $g$ is in $C_{G}(\sigma) V$. This is true since $\sigma$ is semisimple (Corollary $1, \S 6-01$ of [3]). Thus $G=C_{G}(\sigma) R$, whence $G=C_{G}(\sigma)_{0} R$.

For the rest of $\S 3, K$ is an algebraic linear group containing $G$ as a closed normal subgroup; $\sigma$ is a semisimple element of $K$; $G$ acts on $\sigma G$, an element of $h$ of $G$ sending $\sigma g$ into $(\sigma g)^{h}=h^{-1} \sigma g h$ for $g$ in $G$; and if $V$ is a subvariety of $K$, we let $N(V)=N_{G}(V)=\left\{g\right.$ in $\left.G \mid V^{o}=V\right\}$.

Proposition 1. Let $H$ be a Cartan subgroup of $C_{G}(\sigma)_{0}$. Then $N(\sigma H)_{0}$ $=N(\sigma T)_{0}=H$, where $T=H_{s}=\left\{h_{s} \mid h \in H\right\}$.

Proof. We recall that $T$ is the unique maximal torus of $H$ (see [3, §6-04]). Clearly $H \subseteq N(\sigma H)$. And $N(\sigma H) \subseteq N(\sigma T)$ because $(\sigma H)$, $=\sigma H_{s}=\sigma T$ and the action of $G$ on $G$ stabilizes the subset of semisimple elements. It therefore suffices to show that $N(\sigma T)_{0} \subseteq H$. Now $N(\sigma T)=\left\{g \in N(T) \mid g^{\sigma} T=g T\right\}$ (e.g. if $(\sigma T)^{\sigma}=\sigma T$, then $\sigma^{o} T^{o}=\sigma T$ and consequently $\sigma^{o} T=\sigma T$ and $T^{\circ}=T$; the latter implies that $T$ contains $\left(\sigma^{-1}\right)^{\sigma} \sigma=g^{-1} \sigma^{-1} g \sigma$, whence $\left.g T=g^{\sigma} T\right)$. Using this and Lemma 1, we have $N(\sigma T)_{0}=C_{N(\sigma T)_{0}}(\sigma) T \subseteq C_{N(T)_{0}}(\sigma)$. But $N(T)_{0} \subseteq C_{G}(T)[3, \S 6-04]$. Thus $N(\sigma T)_{0} \subseteq\left(C_{G}(T) \cap C_{G}(\sigma)\right)_{0}=H$ (see $\left.[3, \S 7-01]\right)$.

Definition. An element $x$ in $\sigma G$ is $V$-isolated (for $V \subseteq \sigma G$ ) if $x$ is contained in only finitely many distinct sets of the form $V^{o}$ ( $g$ in $G$ ).

Proposition 2. Let $H$ be a Cartan subgroup of $C_{G}(\sigma)_{0}$ and let $T=H_{s}$. Then for some $t$ in $T$, $\sigma t$ is $\sigma T$-isolated and $\sigma H$-isolated. 
Proof. $^{2}$ (a) We first show that if $t$ is an element of $T$ of finite order $m$ relatively prime to $\left(\langle\bar{\sigma}\rangle:(\langle\bar{\sigma}\rangle)_{0}\right)$, then $(\sigma t)^{\circ} \in \sigma H$ implies that $(\sigma\langle t\rangle)^{\circ} \subseteq \sigma T$. Thus suppose that $t$ satisfies the above hypothesis. Then $\left((\sigma t)^{g}\right)^{m}=\left(\sigma^{m} t^{m}\right)^{g}=\left(\sigma^{m}\right)^{g}$ is contained in $\sigma^{m} H$. Thus if $\tau=\sigma^{m}$, it follows that $\left(\tau^{i}\right)^{\circ} \in \tau^{i} H$ for $i$ an integer. Thus $\delta^{-1} \delta^{\circ} \in H$ for $\delta$ in $\langle\tau\rangle$ and therefore for $\delta$ in $\langle\bar{\tau}\rangle$ (since $\delta \rightarrow \delta^{-1} \delta^{\theta}$ is continuous on $K$ and $H$ is closed). Since $\langle\bar{\tau}\rangle \subseteq\langle\bar{\sigma}\rangle$ and $\sigma^{m} \in\langle\bar{\tau}\rangle,\left(\langle\bar{\sigma}\rangle:\langle\bar{\tau}\rangle\right.$ ) divides $m$ and $\langle\bar{\tau}\rangle \supseteq\langle\bar{\sigma}\rangle_{0}$ (for $\bigcup_{i=1}^{m}\langle\bar{\tau}\rangle \sigma^{i}$ is a closed group containing $\sigma$ and thus equals $\left.\langle\bar{\sigma}\rangle\right)$. Since $m$ is relatively prime to $\left(\langle\bar{\sigma}\rangle:\langle\bar{\sigma}\rangle_{0}\right)$, it follows that $\langle\bar{\sigma}\rangle=\langle\bar{\tau}\rangle$ and $\sigma \in\langle\bar{\tau}\rangle$. Thus $\sigma^{-1} \sigma^{\circ} \in H$ and $\sigma^{\sigma} \in \sigma H$.

Since $(\sigma t)^{\sigma}=\sigma^{\sigma} t^{\sigma} \in \sigma H$ and $\sigma^{\sigma} \in \sigma H$, we must have $t^{\sigma} \in H$. Consequently we have $t^{\theta} \in T$. Thus $\langle t\rangle^{\circ} \subseteq T$. Thus $(\sigma\langle t\rangle)^{g} \subseteq \sigma T$, since $\sigma^{\sigma} \in \sigma H$ implies $\sigma^{g} \in \sigma T$.

(b) Let $d=\operatorname{dim} T$. Choose a set $\pi$ of $d$ distinct primes not dividing $\left(\langle\bar{\sigma}\rangle:\langle\bar{\sigma}\rangle_{0}\right)$ or $p$. Then following [3, §6-13], we can find a finite cyclic subgroup $\langle t\rangle$ in $T$ whose order is divisible by a prime $q$ only if $q \in \pi$, and having the property that $\sigma^{-1} g^{-1} \sigma\langle t\rangle g \subseteq T$ if and only if $\sigma^{-1} g^{-1} \sigma T g$ $\subseteq T$. For such a $t, g^{-1} \sigma t g \in \sigma H$ implies that $g^{-1} \sigma\langle t\rangle g \subseteq \sigma T$ by (a), and $g^{-1} \sigma\langle t\rangle g \subseteq \sigma T$ implies that $(\sigma T)^{o}=\sigma T$ by the above choice of $t$. Thus $(\sigma t)^{\circ} \in \sigma H \Leftrightarrow(\sigma T)^{g}=\sigma T$.

We claim that for such a $t, \sigma t$ is $\sigma T$-isolated and $\sigma H$-isolated. $\sigma t$ is $\sigma T$-isolated because $\sigma t \in(\sigma T)^{\sigma}$ implies that $(\sigma t)^{\sigma^{-1}} \in \sigma T \subseteq \sigma H$ which implies that $(\sigma T)^{o}=\sigma T$. We next show that $\sigma t$ is $\sigma H$-isolated. Choose $S \subseteq G$ such that $\sigma t \in(\sigma H)^{s}$ for $s$ in $S$; and such that if $\sigma t \in(\sigma H)^{\circ}$, then $(\sigma H)^{\sigma}=(\sigma H)^{s}$ for a unique $s$ in $S$. We must show that $S$ is finite. If $s$ is in $S$, then $(\sigma t)^{s^{-1}} \in \sigma H$, so that $(\sigma T)^{s^{-1}}=\sigma T$. Thus $S \subseteq N(\sigma T)$. For distinct $r, s$ in $S$, we have $(\sigma H)^{r} \neq(\sigma H)^{s}$ or $r^{-1} s \in N(\sigma H)$. Thus card $S \leqq(N(\sigma T): N(\sigma H)) \leqq\left(N(\sigma T): N(\sigma T)_{0}\right)$ since $N(\sigma H)_{0}=N(\sigma T)_{0}$. Thus $S$ is finite and $\sigma t$ is $\sigma H$-isolated.

Proposition 3. Let $O$ contain a dense open subset of $H$. Then $(\sigma O)^{a}$ contains a dense open subset of $\sigma G$.

Proof. It is easily shown that $(\sigma O)^{G}$ contains an open dense subset of $(\sigma H)^{G}$. And $(\sigma H)^{G}$ contains an open dense subset of $\left[(\sigma H)^{G}\right]^{-}$. Since $N(\sigma H)_{0}=H$ (Proposition 1) and since $\sigma H$ contains a $\sigma H$ isolated point (Proposition 2), $\operatorname{dim}\left[(\sigma H)^{G}\right]^{-}=\operatorname{dim} \sigma H+\operatorname{dim} G$ $-\operatorname{dim} H=\operatorname{dim} G$. (Here we use a very close analog of Lemma 5 of $\S 6-11$ in [3] whose validity is easily seen from the methods of [3, $\S 6-12]$ and Theorem 2 on p. 106 of [2]; details are given in [8, pp.

${ }^{2}$ In this proof we use the misleading notation $\langle\bar{\sigma}\rangle$ for the closed subgroup generated by $\sigma$. 
22-24].) It follows that $\left[(\sigma H)^{G}\right]^{-}=G$ and therefore that $(\sigma O)^{G}$ contains an open dense subset of $G$.

Lemma 2. Let $S=\{t$ in $T \mid \sigma t$ is $\sigma T$-isolated $\}$. Then $S$ contains an open dense subset of $T$.

Proof. Let $B$ denote the set of $\sigma T$-isolated points of $\left[(\sigma T)^{G}\right]^{-}$. Then $B$ contains an open dense subset of $\left.\left[(\sigma T)^{G}\right]\right]^{-}$. (This is also clear from Theorem 2, p. 106 of [2] and the methods of [3]--see corollary of $\S 6-12$; details are given in $[8$, pp. $22-24]$.) Since $(\sigma T)^{G}$ is épais, it follows that $B \cap(\sigma T)^{G}$ contains an open dense subset of $U$ of $\left[(\sigma T)^{g}\right]^{-}$. Let $x$ be an element of $U$. Then $x=(\sigma t)^{g}$ for some $t$ in $T$, $g$ in $G$. Now $\sigma t=x^{\sigma^{-1}} \in U^{\sigma^{-1}} \subseteq B$. Thus $\sigma T \cap U^{\sigma^{-1}}$ is a nonempty open (hence dense) subset of $\sigma T$ which is contained in $\sigma S$.

\section{Lemma 3. Let $\sigma t$ in $\sigma T$ be $\sigma T$-isolated. Then $C_{G}(\sigma t)_{0}=H$.}

Proof. Let $g$ be an element of $C_{G}(\sigma t)$. Then $\sigma t=(\sigma t)^{g} \in(\sigma T)^{\theta}$. Thus the set $S=\left\{(\sigma T)^{\circ} \mid g \in C_{G}(\sigma t)\right\}$ of conjugates of the variety $\sigma T$ is finite, $\sigma t$ being $\sigma T$-isolated. Therefore the isotropy group $C_{G}(\sigma t)$ $\cap N(\sigma T)$ in $C_{G}(\sigma t)$ of the element $\sigma T$ of $S$ is a closed subgroup of $C_{G}(\sigma t)$ of finite index. Thus $C_{G}(\sigma t)_{0} \subseteq C_{G}(\sigma t) \cap N(\sigma T)$. Thus $C_{G}(\sigma t)_{0}$ $\subseteq N(\sigma T)_{0}=H$. On the other hand, it is obvious that $H \subseteq C_{G}(\sigma t)_{0}$.

Proof of TheOREM 1. Regard $G$ as a transformation group on $\sigma G$. Let $T$ be the set of semisimple elements of $H$ and let $S=\{t \in T \mid \sigma t$ is $\sigma T$-isolated $\}$. Let $O_{H}=S U$ where $U$ is the (closed normal connected) subgroup of unipotent elements of $H$ and recall that $s u=u s$ for $s$ in $S$, $u$ in $U[3, \S 6-06, \S 4-07]$. Then $O_{H}$ contains an open dense subset of $H$ by Lemma 2 ; and $H=C_{G}\left(\sigma h_{s}\right)_{0}$ for $h$ in $O_{H}$ by Lemma 3. There is a similar $O_{L}$ for $(\tau, L)$. By Proposition $3,\left(\sigma O_{H}\right)^{G}$ and $\left(\tau O_{L}\right)^{G}$ contain open dense subsets of $\sigma G$. Thus $\left(\sigma O_{H}\right)^{G} \cap\left(\tau O_{L}\right)^{G}$ is nonempty and there exist $g_{1}, g_{2}$ in $G, h$ in $O_{H}, l$ in $O_{L}$, such that $g_{1}{ }^{-1} \sigma h g_{1}=g_{2}{ }^{-1} \tau l g_{2}$. Then $g_{1}{ }^{-1} \sigma h_{s} g_{1}=g_{2}{ }^{-1} \tau l_{s} g_{2}$. Taking connected centralizers in $G$, we have $g_{1}^{-1} H_{1}=g_{2}{ }^{-1} L g_{2}$. The assertions of the theorem follow immediately.

4. Fixed points and stable subgroups of $\sigma$. The following theorem is shown in [7] to be a consequence of the preceding theorem:

Theorem 2. Let $G$ be a connected semisimple algebraic group. Let $\sigma$ be a semisimple (algebraic) automorphism of $G$. Then $\sigma$ keeps stable a Borel subgroup $B$ of $G$ and a maximal torus $T$ of $G$ contained in $B$. For any such $B$ and $T, F_{T}(\sigma)_{0}$ is a Cartan subgroup of $F_{G}(\sigma)_{0} . F_{G}(\sigma)_{0}$ contains $a$ regular element of $G$. 
TheOREM $3 .^{3}$ Let $\sigma$ be a semisimple algebraic automorphism of a connected algebraic group $G$. Then $\sigma$ keeps stable a Borel subgroup of $G$. Moreover the centralizer in $G$ of a maximal torus in $F_{G}(\sigma)_{0}$ is solvable.

Proof. Let $R$ be the radical of $G$. Then $\sigma$ induces an automorphism $\bar{\sigma}$ on $\bar{G}=G / R . \bar{\sigma}$ is clearly a semisimple algebraic automorphism of $\bar{G}$. By Theorem 2, $\bar{\sigma}$ stabilizes a Borel subgroup of $\bar{G}$. The latter must have the form $\bar{B}=B / R$ where $B \supseteq R$ and $B$ is a $\sigma$-stable subgroup of $G$.

A maximal torus of $F_{\bar{G}}(\bar{\sigma})_{0}$ has the form $\bar{C}=C / R$ where $C \geqq R$. By Lemma $1, C=F_{C}(\sigma)_{0} R$. Since $F_{C}(\sigma)_{0}$ is solvable, $F_{C}(\sigma)_{0}=S U$ where $S$ is a torus and $U$ is a closed connected unipotent subgroup. Since $C / R$ is a torus in $\bar{G}, U \subseteq R$. Thus $C=S R$. By Theorem $8, F_{\bar{G}}(\bar{\sigma})_{0}$ contains a regular element of $\bar{G}$. Thus the same is true of $\bar{C}$ and $C_{\bar{G}}(\bar{C})$ is abelian. (The centralizer of a torus in a connected algebraic group is connected $[3, \S 6-14]$.) Thus $C_{G}(S)$ is solvable (since $C_{G}(S) R / R \subseteq C_{\bar{G}}(\bar{C})$ ). This establishes that the centralizer of a maximal torus in $F_{G}(\sigma)_{0}$ is solvable, since any such torus contains a conjugate of $S$.

Lemma 4. Let $G$ be a connected solvable algebraic group. Let $\mathfrak{g}$ be a finite group of birational automorphisms of $G$. Then if $g$ has order relatively prime to $p, g$ keeps stable a Cartan subgroup of $G$.

Proof. Let $G=T U$ be the usual decomposition of $G$ as product of a torus $T$ and a closed connected characteristic unipotent subgroup $U$. $U$ is $g$-stable. An easy induction argument shows that we can assume without any loss of generality that $U$ is abelian and that $C_{U}(G)_{0}$ $=\{1\}$. Since $G$ is solvable, $N_{U}(T)=C_{U}(T)$ and both are connected $[3, \S 6-04]$. Since $U$ is abelian, $C_{u}(T)=C_{U}(G)$. Thus $N_{U}(T)=N_{U}(T)_{0}$ $=C_{U}(G)_{0}=\{1\}$ and $N_{U}(T)=\{1\}$. Thus for $\sigma$ in $\mathfrak{g}$, there is a unique $u_{\sigma}$ in $U$ such that $T^{\sigma}=T^{u_{\sigma}}$. The mapping $u_{\sigma}$ from $\mathfrak{g}$ in to $U$ is clearly a 1 -cocycle. Since $U$ is uniquely $m$-divisible where $m$ is the order of $\mathfrak{g}$, $H^{1}(\mathfrak{g}, U)=\{0\}$ and there exists $v$ in $U$ such that $v\left(v^{-1}\right)^{\sigma}=u_{\sigma}$ for $\sigma$ in $\mathfrak{g}$. It is seen by a simple computation that $T^{v}$ is $\mathfrak{g}$-stable. Thus so is the Cartan subgroup $C(T)^{v}$.

TheOREM 4. Let $\sigma$ be a semisimple algebraic automorphism of a connected algebraic group $G$. Then $\sigma$ keeps stable a Cartan subgroup of $G$.

Proof. By Theorem 3, $\sigma$ keeps stable a Borel subgroup of $G$. Thus with no loss of generality we may assume that $G$ is solvable. We may assume that $G$ is a closed connected normal subgroup of $K$ and that $s$ is a semisimple element of $K$ such that $\sigma=\operatorname{Int}_{G} s$. Let $S$ be the closed

${ }^{3}$ R. Steinberg has proved independently, and under weaker conditions on $\sigma$, that $\sigma$ stabilizes a Borel subgroup of $G$. 
subgroup generated by $s$. Then $S$ is diagonalizable and $S=\mathrm{g} S_{0}$ for some finite subgroup $g$ of $S$ (e.g. see part of the proof of Lemma 1). Now the connected centralizer of $S_{0}$ in $\mathfrak{g}$ has a $\mathfrak{g}$-stable maximal torus $T$ by Lemma 4. Clearly $S_{0} \subseteq T$ and therefore $T$ is a maximal torus of $G$. $T$ is clearly $S$-stable. Thus $T$ is $\sigma$-stable. Thus $\sigma$ stabilizes the Cartan subgroup $C(T)$.

\section{BIBLIOGRAPHY}

1. A. Borel and G. D. Mostow, On semi-simple automorphisms of Lie algebras, Ann. of Math. 61 (1955), 389-504.

2. C. Chevalley, Fondements de la géométrie algébrique, Faculté des Sciences de Paris, 1958.

3. C. Chevalley (Séminaire C. Chevalley), Classification des groupes de Lie algêbrique, Vols. I, II, École Norm. Sup., Paris, 1958.

4. G. Hochschild and G. D. Mostow, Representations and representative functions of Lie groups, Ann. of Math. 66 (1957), 495-542.

5. N. Jacobson, A note on automorphisms of Lie algebras, Pacific J. Math. 12 (1962), 303-315.

6. G. D. Mostow, Fully reducible subgroups of algebraic groups. Amer. J. Math. 78 (1956), 200-221.

7. D. J. Winter, On automorphism of algebraic groups, Bull. Amer. Math. Soc. 72 (1966), 706-708.

8. - On automorphisms of Lie algebras and algebraic groups, Doctoral Dissertation, Yale Univ., New Haven, Conn., 1966.

YALE UNIVERSITY 